\title{
EXTENDED DEPTH-OF-FOCUS FOR MULTI-CHANNEL MICROSCOPY IMAGES: A COMPLEX WAVELET APPROACH
}

\author{
Brigitte Forster, Dimitri Van De Ville, Jesse Berent, Daniel Sage, Michael Unser
}

\author{
Swiss Federal Institute of Technology Lausanne (EPFL) \\ Biomedical Imaging Group, CH-1015 Lausanne, Switzerland
}

\begin{abstract}
Microscopy imaging often suffers from limited depth-offocus. However, the specimen can be 'optically sectioned' by moving the object along the optical axis; different areas appear in focus in different images. Extended depthof-focus is a fusion algorithm that combines those images into one single sharp composite. One promising method is based on the wavelet transform. In this paper, we show how the wavelet-based image fusion technique can be improved and easily extended to multi-channel data. First, we propose the use of complex-valued wavelet bases, which seem to outperform traditional real-valued wavelet transforms. Second, we introduce a way to apply this technique for multi-channel images that suppresses artifacts and does not introduce false colors, an important requirement for multi-channel fluorescence microscopy imaging. We evaluate our method on simulated image stacks and give results relevant to biological imaging.
\end{abstract}

\section{INTRODUCTION}

Limited depth-of-focus is a common problem in biological imaging with conventional light microscopy. Often, the specimen turns out to be thicker than the attainable focal depth. Portions of the object's surface outside the focal plane appear defocused in the acquired image plane. This becomes worse as the magnification increases because the numerical aperture increases, too, and therefore the depth-of-focus becomes smaller. Consequently, each acquisition will be compromised and show certain parts of the specimen in and out of focus. To image the whole specimen, multiple images are taken at different focal planes. The challenge then becomes to select from each slice the area that is focused in order to reconstruct an image that is sharp everywhere. In this way, it is possible to extend the depth of focus without the physical limitation of the numerical aperture of the objective lens (see Fig. 1).

Numerous articles have been written on this matter and some solutions have proven to be more efficient than others. An overview can be found in [1-3]. One of the known methods is based on the wavelet transform, for which we propose two extensions in this paper. Basically, our approach computes the discrete complex-valued

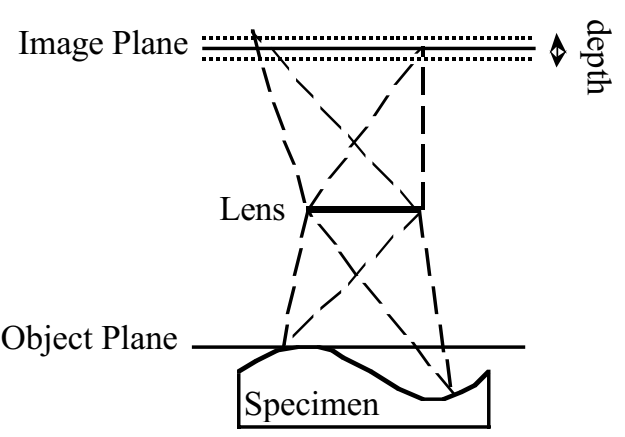

Fig. 1. Microscopic imaging system, depth-of-focus.

wavelet transform of each image slice of the object, and builds up the wavelet coefficients of the composite image by a maximum-absolute-value selection rule. The final composite image is obtained after computing the inverse complex wavelet transform. For successful application to multi-channel images, we propose an adapted multichannel conversion and recovery as a pre- and postprocessing step. In essence, this method compensates for the increase in dynamic range of the image obtained after fusion and avoids the introduction of false colors [4]. We propose a quantitative validation procedure that allows us to compare our method with other approaches and to evaluate its performance. We also present results obtained with real specimens.

\section{EXTENDED DEPTH-OF-FOCUS USING THE WAVELET TRANSFORM}

To extend the depth-of-focus, we first have to define an in-focus criterion. Typically, an image that is in-focus has a maximal number of visible details. On the other hand, defocused images are blurred by the point-spread-function of the microscope. Therefore, we suppose that the areas of an image that are focused contain more high frequency components than out-of-focus areas.

Classical frequency analysis, using the Fourier transform, does not provide any spatial localization. The wavelet transform, by contrast, seems to be the ideal high saliency detection, since it allows a local analysis of the image's frequency content. Unlike windowed Fourier transforms, this type of multi-resolution automatically adapts to 
various sizes of details. Last but not least, it provides perfect reconstruction and preserves the size of the images.

\section{IMAGE FUSION ALGORITHM}

The image fusion algorithm proposed in this paper relies on two conditions prior to processing. The first is that the images must be aligned throughout the stack and the second is that they must be at the same magnification level. Both conditions are met if the original images are taken by a traditional light microscope with the specimen being translated along the optical axis $z$ to obtain the slices.

\subsection{Complex-valued Wavelet Transform}

The discrete wavelet transform was proposed for image fusion in many papers (for example, see [2] for an overview). The proposed wavelet transforms are bi- or orthonormal basis decompositions and thus the information in different bands is unique. Moreover, it allows perfect reconstruction and fast implementations [7]. However, not all wavelets have the same performance. We choose the complex Daubechies wavelets invented independly by Lawton [5] and by Lina and Mayrand [6] They perform a complex-valued wavelet transform by filtering with finite-length complex low and high pass filters. They provide complex wavelet bases of various degrees and thus an adjustment of the order of smoothing performed by the transform is possible. Here, we selected the complex Daubechies wavelets with four vanishing moments and 6/6 taps filters, because they provide an orthonormal decomposition, which is useful for an eventual additional denoising step, and because they perform an adequate smoothing.

For each slice $s(., . ; z)$ of the stack $\{s(x, y ; z)\}_{z}$, we perform a 2D separable complex wavelet transform CWT

$$
C W T: s(x, y ; z) \rightarrow \quad\left\{c_{j}(n, m ; z)\right\}_{j}
$$

and obtain complex-valued coefficients $c_{j}$ for each scale $j$. We found that the complex-valued transform adds robustness to the selection rule and consistency check algorithms.

\subsection{Selection Rule and Consistency Checks}

The largest absolute value of the coefficients in the subbands will correspond to sharper brightness changes and therefore to the most salient features. A good integration rule consists in selecting the slice with the largest absolute value of the wavelet coefficients at each point:

$$
d_{j}(n, m)=c_{j}\left(n, m ; \arg \max _{z}\left|c_{j}(n, m ; z)\right|\right) .
$$

The fused transform based on the combined complex coefficients can thereafter be inverted in order to obtain a composite image. We store the number of the selected slice per pixel in a separate map. It may be used for consistency checks or topological visualization.

There are several approaches on consistency checks for the choice of the coefficients. The two most important are
- Spatial consistency: The majority of neighboring pixels in a $3 \times 3$ window shall be chosen from the same slice.

- Subband consistency: Corresponding pixels in different subbands shall be chosen from the same slice.

\subsection{Inverse Complex Discrete Wavelet Transform}

We compute the inverse complex wavelet transform on the fused coefficient matrix to get an intermediate fused image:

$$
C W T^{-1}:\left\{d_{j}(n, m)\right\}_{j} \rightarrow \quad p(x, y) .
$$

In general, the pixels of the reconstructed image are complex numbers. We considered the transformation on real values by calculating the absolute value of the complex number per pixel and by just taking the real parts. We found that the second approach performs better.

\section{PRE- AND POST-PROCESSING STEPS}

\subsection{Pre-processing: Multi-channel conversion}

Using the fusion method based on the wavelet transform, one would be tempted to apply the algorithm to multichannel data, by treating each channel independently. Such an approach could be useful for multi-channel data where the details are not correlated across channels. However, if the channels jointly contribute to details, this method will cause saturation and false colors. Moreover, we would have to perform the whole algorithm several times, which multiplies the computation time and storage space. We propose a method to convert color images to greyscale in order to apply the algorithm once.

The traditional method to perform color conversion from RGB images to grayscales consists in a fixed weighted average of the three colors (i.e., $\mathrm{Y}=0.30 \mathrm{Red}+$ 0.59 Green +0.11 Blue). This is a good universal colour to greyscale transformation in particular for natural images. However, in microscopy images, a particular color might be highly present (e.g., a specimen stained using a red colorant). Therefore, we adapt the weights to the different channels $k$ according to their average intensity:

$$
\left\{s^{(k)}(x, y ; z)\right\}_{k} \rightarrow \quad\{s(x, y ; z)\} .
$$

In this way, a predominant color will be given more weight during the subsequent fusion algorithm.
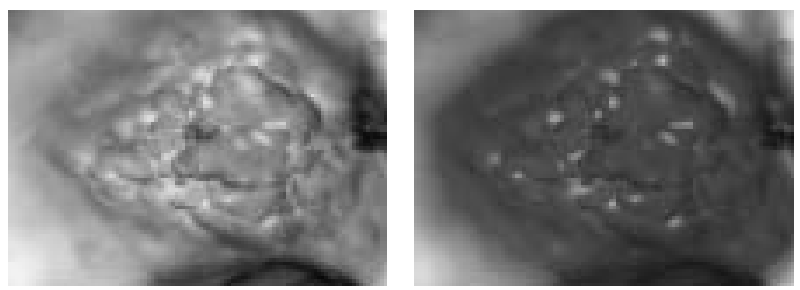

Fig. 2. One slice of a stack showing Peyer plaques from the intestine of a mouse. Multi-channel conversion with (left) and without (right) adaptive weights. 
The greyscale images created preserve more saliency than by simply using fixed weights (see Fig. 2).

\subsection{Post-Processing: Clipping}

Fusion in the wavelet domain yields results that are "non convex" combinations of the pixel values of the input images; a consequence of retaining the maximal absolute value coefficient. For typical images, approximately $30 \%$ of the pixels are over or under the original dynamic range of all images. Such an increase of dynamic range boosts the global energy of the image, the noise level, and the amount of saturation.

To at least avoid the non-convexity errors, we propose a "clipping" algorithm that selects the closest available value according to the original data. Multi-channel clipping for each channel $k$ can be expressed as

$$
q^{(k)}(x, y)=s^{(k)}\left(x, y ; \arg \min _{z}|p(x, y)-s(x, y ; z)|\right) .
$$

This procedure eliminates outliers and diminishes ringing effects (see Fig. 3). Moreover it can be applied to "restore" the multi-channel pixel data, without false color effects, by construction.
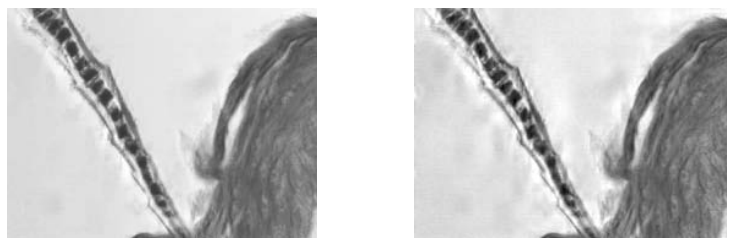

Fig. 3. Mouse epidermis. Clipping (left) diminishes ringing effects (right).

\section{COMPARISON WITH OTHER METHODS}

To evaluate our method we simulated microscopical data and compared the reconstructed image with the ground truth. We compared our complex wavelets method with the real wavelets and the variance method.

\subsection{Classical approach}

The variance method is based on the assumption that in focus regions of the image correspond to high variations of intensity. The variance over a $3 \times 3$ window is calcultated in order to determine whether the center pixel is in a focused area. The pixel along the z-axis with the highest variance is chosen for the composite image. This algorithm has the advantage of computational simplicity.

\subsection{Evaluation}

Four simulated stacks are constructed as illustrated in Fig. 4. Brodatz textures (D18, D22, D23, D112) are mapped onto a surface and discretized using linear interpolation along the $z$-direction. Next, the volume is convolved by a filter that contains a Gaussian blur for each slice with increasing width as the defocus increases. We compared the variance method, the real wavelet method and the complex wavelet method. We used the real orthonormal spline wavelet of degree 3 and the

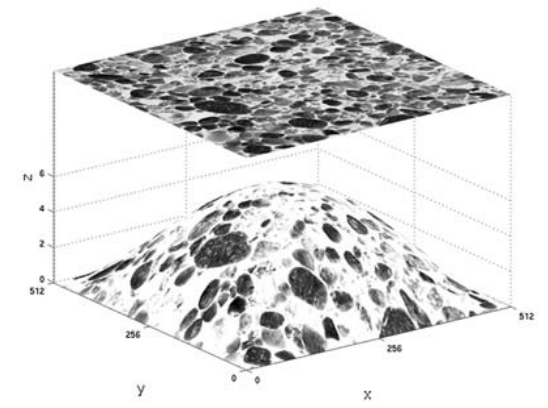

Fig. 4. Construction of a simulated data stack by projecting texture on a surface.

6/6 tap complex Daubechies wavelet, since they have the same number of vanishing moments. Moreover, we evaluated the influence of clipping and of the consistency checks. For all experiments we measured the SNR with respect to the ground truth. The results are given in Tab. 1. We found that both wavelet methods outperform the variance method. The quality of the output image of the variance method is quite poor. Problems especially occur when the focused area contains homogenous parts, and often artificial details are introduced.

Comparing complex and real wavelets, the complex ones give us about $0.7 \mathrm{~dB}$ performance gain. Clipping adds about $0.3 \mathrm{~dB}$ for both wavelet methods. Consistency checks improve the quality of the result, especially when they are combined with clipping. However, the consistency checks turned out to be very costly with respect to storage space and computation time. For the processing of real microscopic stacks (typical resolution of $1996 \times$ 1450 , about 40 images) we therefore set them aside and perform the complex wavelet method with clipping.

\subsection{Results}
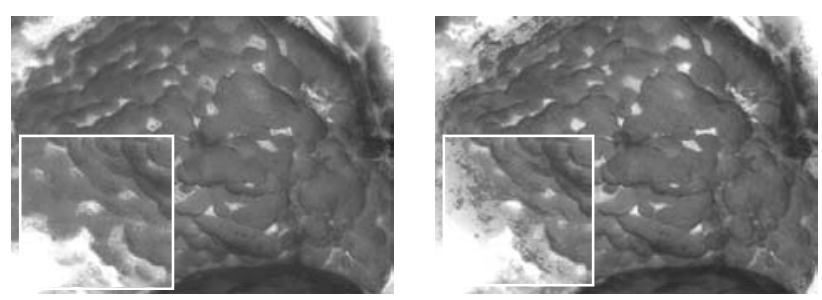

Fig. 5.a. The variance method (left), and the real wavelet method (right) with clipping.

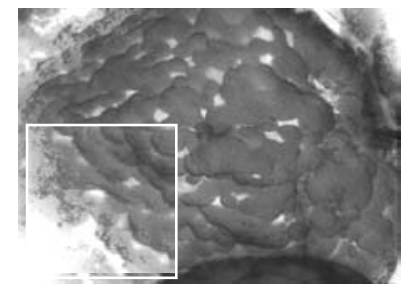

Fig. 5.b. The complex wavelet method with clipping. 


\begin{tabular}{|lcc|c|}
\hline $\begin{array}{l}\text { Performance gain } \\
\text { compared to } \\
\text { Variance Method }\end{array}$ & Spline 3 & CDaub 6 & $\begin{array}{c}\text { CDaub 6 } \\
\text { comp. to } \\
\text { Spline 3 }\end{array}$ \\
\hline No manipulations & $2.49 \mathrm{~dB}$ & $3.21 \mathrm{~dB}$ & $0.72 \mathrm{~dB}$ \\
Clipping & $2.87 \mathrm{~dB}$ & $3.51 \mathrm{~dB}$ & $0.64 \mathrm{~dB}$ \\
Spatial Check & $3.55 \mathrm{~dB}$ & $3.67 \mathrm{~dB}$ & $0.12 \mathrm{~dB}$ \\
Spatial Check, Clipping & $3.74 \mathrm{~dB}$ & $3.89 \mathrm{~dB}$ & $0.15 \mathrm{~dB}$ \\
Subband Check & $4.14 \mathrm{~dB}$ & $4.50 \mathrm{~dB}$ & $0.36 \mathrm{~dB}$ \\
Subb. Ch., Clipping & $4.33 \mathrm{~dB}$ & $4.70 \mathrm{~dB}$ & $0.37 \mathrm{~dB}$ \\
\hline
\end{tabular}

Tab. 1. The average performance gain of the real and the complex wavelet method with preprocessing and consistency checks compared to the variance method. The right column compares the complex wavelet method with the real one. The SNRs of the wavelet methods range between $23 \mathrm{~dB}$ and $29 \mathrm{~dB}$.

We discuss our results on the example of Peyer plaques from the intestine of a mouse (see Fig. 5 a, b). The specimen is strongly stained with a red colorant.

Also for real data the complex wavelet method with clipping proposed in this paper outperforms the classical fusion method. It gives more details in the boundary regions, but does not introduce artificial details.

\section{SOFTWARE PACKAGE}

We implemented our method in Java as an ImageJ plugin. ImageJ is a widely used multi-platform public domain software for the analysis of biological images. Our plug-in can be downloaded from our web site

$$
\text { http://bigwww.epfl.ch/demo/edf/ }
$$

It allows the fusion of a stack of images by just one single mouse click, see Fig. 6.

\section{CONCLUSIONS}

We presented a new method to extend the depth-of-focus of microscopic images. By measurements on simulated data and by examples on real images, we showed that our choice of a complex wavelet transform outperforms real wavelets and the variance method. We showed that a careful multi-channel conversion with subsequent clipping avoids the introduction of false colors, and suppresses artifacts. Our algorithm is freely available as a plug-in for ImageJ and is used in practice by biologists at the ISREC cancer research facility in Lausanne. The algorithm can be applied to visualize and present light microscopy images. Another application includes the reconstruction of a sharp DIC (differential interference contrast) image, containing structural information of a biological specimen, that is superposed with fluorescence images [8].

In the future, we will incorporate non-linear denoising in the wavelet domain and work on improving the height map for 3D reconstruction.

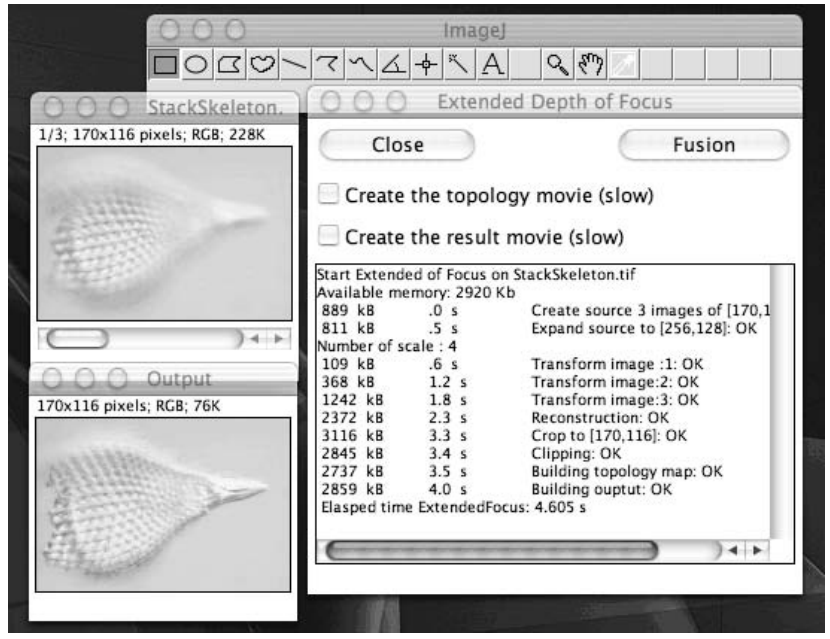

Fig. 6. Screenshot of our software package, which is freely available as a plug-in for the ImageJ Java Image Processing Framework.

\section{ACKNOWLEDGEMENTS}

The images are courtesy of Jelena Mitic at LOB/EPFL, Zeiss, and MIM at ISREC Lausanne. The first author acknowledges the financial support from the German Academy of Natural Scientists Leopoldina (BMBF-LPD 9901/8-64) and from the European Union's Human Potential Program (HPRN-CT-2002-00285).

\section{REFERENCES}

[1] H. Li, B.S. Manjunath, and S.K. Mitra, "Multisensor Image Fusion Using the Wavelet Transform", Graphical Models and Image Processing, Academic Press, Vol. 57, No. 3, May, pp. 235-245, 1995.

[2] A.G. Valdecasas, D. Marshall, J.M. Becerra, and J.J. Terrero, "On the extended depth of focus algorithms for bright field microscopy”, Micron, Elsevier, Vol. 32, pp. 559-569, 2001.

[3] A.G. Valdecasas, D. Marshall, and J.M. Becerra, "Extended Depth-of-focus Algorithms in Brightfield Microscopy", European Microscopy and Analysis, Rolston Gordon Communications, Vol. 79, pp. 15-17, September 2002.

[4] J. Berent, "Extended depth of focus through image fusion using the wavelet transform", Technical Report, LIB, EPF Lausanne, June 2003.

[5] W. Lawton, "Applications of complex valued wavelet transforms to subband decomposition", IEEE Transactions on Signal Processing, IEEE, Vol. 41, no. 12, pp. 3566-3568, 1993.

[6] J.-M. Lina and M. Mayrand, "Complex Daubechies Wavelets", Applied and Computational Harmonic Analysis, Academic Press, Vol. 2, p. 219-229, 1995.

[7] S. Mallat, A Wavelet Tour of Signal Processing, Academic Press, 1998.

[8] F. Kagalwala and T. Kanade, "Reconstructing Specimens Using DIC Microscope Images", IEEE Tr. on Systems, Man, and Cybernetics-Part B: Cybernetics, IEEE, Vol. 33, No. 5, pp. 728-737, October 2003. 